\title{
Packing non-returning A-paths algorithmically
}

\author{
Gyula $\operatorname{Pap}^{1 \dagger}$ \\ ${ }^{1}$ Dept. of Operations Research, Eötvös University, Pázmány P. s. 1/C, Budapest, Hungary H-1117.
}

\begin{abstract}
In this paper we present an algorithmic approach to packing $A$-paths. It is regarded as a generalization of Edmonds' matching algorithm, however there is the significant difference that here we do not build up any kind of alternating tree. Instead we use the so-called 3-way lemma, which either provides augmentation, or a dual, or a subgraph which can be used for contraction. The method works in the general setting of packing non-returning $A$-paths. It also implies an ear-decomposition of criticals, as a generalization of the odd ear-decomposition of factor-critical graph.
\end{abstract}

Keywords: $A$-paths, matching

\section{Introduction}

The paper is devoted to the problem of packing fully node-disjoint non-returning $A$-paths in a graph $G=(V, E)$. Given a graph and a subset $A \subseteq V$, a path is said to be an $A$-path if its ends are two distinct nodes in $A$. Packing fully node-disjoint $A$-paths reduces to maximum matching in an auxiliary graph, see T. Gallai (3). The special case $A=V$ is in fact equivalent to maximum matching. W. Mader considered a more difficult problem. We are given a subset $A \subseteq V$ with a partition $\mathcal{A}$. An $A$-path is called an $\mathcal{A}$ path if its ends are in two distinct members of $\mathcal{A}$. Mader (5) gave a min-max formula for the maximum number of fully node-disjoint $\mathcal{A}$-paths. A polynomial time algorithm to find these paths was given by L. Lovász using his matroid parity apparatus. Matroid parity is still a challanging topic in combinatorial optimization. If a problem turns out to be an instance for matroid parity, this does not necessarily imply a polynomial time algorithm or a good characterization. Lovász disentangled some techincal details to construct an algorithm, see (4). Later, A. Schrijver gave a funny reduction to linear matroid parity which by itself also implies an algorithm. It was a challange to construct directly an algorithm for packing $\mathcal{A}$-paths. Such an algorithm was given by Chudnovsky et al. (2). They in fact work with the concept of non-zero $A$-paths, which is a generalization of $\mathcal{A}$-paths, see also (1). The main goal of this paper is to construct an algorithm which presents the "dual" in a more structured form. Our method implies an ear-decomposition of "criticals" - this generalizes the ear-decomposition of factor-critical graphs.

Maximum matching is a special case of the problem discussed in this paper, let us briefly sketch how the method works for maximum matching. For a given matching $M \subseteq E$ in $G$, we call an odd cycle $C \subseteq E$ an $M$-alternating odd cycle if $|C \cap M|=(|C|-1) / 2$ and $C$ is incident to an $M$-exposed node. The following lemma can be proved directly, a proof "on the level of bipartite matching" can be given. In

\footnotetext{
${ }^{\dagger}$ Research is supported by OTKA grants T 037547 and TS 049788, by European MCRTN Adonet, Contract Grant No. 504438 and by the Egerváry Research Group of the Hungarian Academy of Sciences. e-mail: gyuszko@cs.elte. hu
} 
fact, Edmonds' alternating forests provide an alternative proof of this lemma. Our crucial observation is that a matching algorithm can be constructed by only using the below lemma as a black box. This black box is regarded as a compact formulation of some consequences of alternating forests. However, one can also give a short, inductive proof without alternating forests.

Lemma 1.1 (3-Way Lemma for Matching) Given an undirected graph $G$ with a matching $M$, then at least one of the following alternatives holds:

1. There is a matching $N$ with $|N|=|M|+1$.

2. There is a matching $N$ with $|N|=|M|$ and an $N$-alternating odd cycle in $G$.

3. There is a vector $c \in\{0,1,2\}^{V}$ such that the weight of any edge is at least 2 , and the sum of its entries is exactly $2|M|$.

This lemma allows us to interpret of Edmonds' algorithm as follows. Consider a matching $M$ in graph $G$, try Lemma 1.1. Alternative 1 gives an augmentation, alternative 3 verifies optimality. Alternative 2 provides an odd cycle for contraction. Contraction of an alternating odd cycle has the property that augmentation, or a Berge-Tutte-dual in $G / C$ can be expanded to $G$.

\section{Packings in p-graphs - Definitions}

The most important notion in this paper is a permutation labeled graph or p-graph, for short. A p-graph comes in the form of $G, A, \omega, \pi$, where $G$ is a graph, $A$ is a set of nodes, $\pi$ are edge-labels. This notion provides a generalization of some well-known packing problems - matching, node-disjoint $\mathcal{A}$-paths, nonzero $A$-paths. The motivation for this version is that important reduction principles used by our algorithm stay within the concept of a p-graph, but does not stay within well-known previous concepts. The precise definition of a p-graph is formulated as follows.

Let $G=(V, E)$ be an undirected graph with node-set $V$, edge-set $E$ with a reference orientation. Let $A \subseteq V$ be a fixed set of terminals. Let $\Omega$ be an arbitrary set of "potentials" and let $\mathbf{j j}$, $\mathbf{J J}$ be called Jolly Joker (some imaginary labels). Let $\omega: A \rightarrow \Omega$ define the potential of origin for the terminals. Let $\pi: E \rightarrow S(\Omega) \cup\{\mathbf{J J}\}$ where $S(\Omega)$ is the set of all permutations of $\Omega$. For an edge $a b=e \in E$, let $\pi(e, a):=\pi(e)$ and $\pi(e, b):=\pi^{-1}(e)$ be the mapping of potential on edge $a b$. (We use $\circ$ for the composition of permutations. We define $\mathbf{J J}^{-1}:=\mathbf{J} \mathbf{J} \circ \pi:=\pi \circ \mathbf{J J}:=\mathbf{J} \mathbf{J}$ and $\mathbf{J J}(\omega):=\pi(\mathbf{j} \mathbf{j}):=\mathbf{j j}$ for any $\pi \in S(\Omega) \cup\{\mathbf{J J}\}$ and for any $\omega \in \Omega \cup\{\mathbf{j} \mathbf{j}\}$.) A walk in $G$ is a sequence of nodes and edges, say $W=\left(v_{0}, e_{0}, v_{1}, e_{1}, \ldots, e_{k-1}, v_{k}\right)$ where $e_{i}=v_{i} v_{i+1}$ or $e_{i}=v_{i+1} v_{i}$ for all $0 \leq i \leq k-1 . W$ is called an $A$-walk in $G$ if $v_{0}, v_{k} \in A$ and $v_{j} \notin A$ (for $j \neq 0, k$ ). $\chi_{W} \in \mathbb{N}^{V}$ denotes the traversing multiplicity vector of walk $W$, defined by $\chi_{W}(v):=\left|\left\{j: v_{j}=v\right\}\right|$. A walk $W$ is called a path if $\chi_{W} \leq \mathbf{1}$. We will usually use letters $P, R$ for paths. For an $A$-walk let $\pi(W):=\pi\left(e_{0}, v_{0}\right) \circ \pi\left(e_{1}, v_{1}\right) \circ \ldots \circ \pi\left(e_{k-1}, v_{k-1}\right)$ define the mapping of potentials on $W$. $W$ is called non-returning if $\pi(W)\left(\omega\left(v_{0}\right)\right) \neq \omega\left(v_{k}\right)$. (Hence, an empty $A$-walk (having a single node and no edge) is not considered to be non-returning. Notice, if $W$ traverses any edge with label $\mathbf{J J}$, then $W$ is non-returning.) A family $\mathcal{P}$ of fully node-disjoint nonreturning $A$-paths is called a packing. $\nu=\nu(G)=\nu(G, A, \omega, \pi)$ denotes the maximum cardinality of a packing. Also, a "node-capacited packing problem" can be defined. Consider a function $b \in \mathbb{N}^{V}$ of node capacities. A family $\mathcal{W}$ of $A$-walks (we allow walks to be taken multiply) is called a $b$-packing if $\sum_{W \in \mathcal{W}} \chi_{W} \leq b$. Let $\nu_{b}=\nu_{b}(G)=\nu_{b}(G, A, \omega, \pi)$ denotes the maximum cardinality of a $b$-packing. $b=\mathbf{1}$ defines packings, $b=\mathbf{2}$ defines 2-packings. 


\section{Min-max Theorems for packings}

For a set $F \subseteq E$ of edges, let $A^{F}:=A \cup V(F) . F$ is called $A$-balanced if $\omega$ can be extended to a function $\omega^{F}: A^{F} \rightarrow \Omega$ s.t. each edge $a b \in F$ is $\omega^{F}$-balanced - i.e. $\pi(a b, a)\left(\omega^{F}(a)\right)=\omega^{F}(b)$. Let $c_{\text {odd }}(G, A)$ be the number of components in $G$ having an odd number of nodes in $A$ - these will be called odd components of $G, A$. Let $c_{1}(G, A)$ be the number of nodes in $A$ which are isolated nodes of $G$.

Theorem 3.1 In a p-graph the maximum cardinality of a packing is determined by

$$
\nu(G, A, \omega, \pi)=\min _{F, X}|X|+\frac{1}{2}\left(\left|A^{F}-X\right|-c_{o d d}\left(G-F-X, A^{F}-X\right)\right),
$$

where the minimum is taken over an A-balanced edge-set $F$ and a set $X \subseteq V$.

Theorem 3.2 In a p-graph the maximum cardinality of a 2-packing is determined by

$$
\nu_{2}(G, A, \omega, \pi)=\min _{F, X} 2|X|+\left|A^{F}-X\right|-c_{1}\left(G-F-X, A^{F}-X\right),
$$

where the minimum is taken over an A-balanced edge-set $F$ and a set $X \subseteq V$.

In Theorem 3.2 we do not count odd components to determine a maximum 2-packing, this indicates that 2-packings are simpler than packings. A similar relation there is between matchings and 2-matchings, the latter admitting a reduction to bipartite matching, König's Theorem. The following theorem is in fact a reformulation of Theorem 3.2, here we formulate a Kőnig-type condition for 2-packings.

Theorem 3.3 In a p-graph the maximum cardinality of a 2-packing is determined by

$$
\nu_{2}(G, A, \omega, \pi)=\min \|c\|,
$$

where $\|c\|:=\sum_{v \in V} c(v)$ and the minimum is taken over 2-covers $c$, i.e. vectors $c \in\{0,1,2\}^{V}$ such that $c \cdot \chi_{W} \geq 2$ for any non-returning $A$-walk.

\section{Contraction of dragons}

A path $P$ is called a half- $A$-path if it starts in a terminal $s \in A$, ends in a node $t \in V$ and $V(P) \cap A=\{s\}$. We say $P$ ends in $t$ with potential $\pi(P)(\omega(s))$. Consider a node $v \in V$ and a potential $\omega_{0} \in \Omega \cup\{\mathbf{j j}\}$. We say a node $v$ is $\omega_{0}$-reachable (or $\omega_{0}$ is reachable at $v$ ), if there is a pair $\mathcal{P}, P_{v}$ such that $P_{v}$ is a half- $A$-path ending in $v$ with $\omega_{0}$, and $\mathcal{P}$ is a packing of $\nu$ non-returning $A$-paths each of which is fully node-disjoint from $P_{v}$. We say a node is reachable if it is $\omega_{0}$-reachable for some $\omega_{0} \in \Omega \cup\{\mathbf{j j}\} . v$ is called uniquely reachable if it is $\omega_{0}$-reachable only with a single element $\omega_{0} \neq \mathbf{j j}$. Otherwise - if $v$ is jj-reachable or there are at least two different elements of $\Omega$ which are reachable at $v$, then $v$ is called multiply reachable. The definition implies that a reachable terminal is uniquely reachable. We call a p-graph $G$ a dragon if $|A|=2 \nu+1$ and every node is reachable. A p-graph is called critical if it is a dragon such that every non-terminal is multiply reachable. (The notion of criticals is analogue to the notion used in (1). The notion of dragons should be considered as a weak version of criticality.) Let us use the expression odd cycle for p-graphs s.t. $G=(V, E)$ is an odd cycle, $A=V$, and all the edges in $E$ give one-edge non-returning $A$-walks (which are in fact non-returning $A$-paths except for 1-edge odd cycles). A p-graph with $V=\{a, b\}, E=\{a b\}, A=\{a\}$ is called a rod. 
Claim 4.1 Odd cycles and rods are dragons.

A crucial lemma is the following, saying that the min-max formula holds for dragons.

Lemma 4.2 (A dragon has a special dual) Suppose a $G$ is a dragon with exactly its nodes in $V_{1}$ being uniquely reachable, say $v \in V_{1}$ is $\omega^{\prime}(v)$-reachable. Let $F:=\left\{e \in E\left[V_{1}\right]: e\right.$ is $\omega^{\prime}$-balanced $\}$. Then $2 \nu=\left|V_{1}\right|-c\left(G-F, V_{1}\right)$.

The notion "reachability" is in fact motivated by the goal to define the contraction of dragon subgraphs.

Definition 4.3 (Contraction of a dragon) Consider a set $Z \subseteq V$ such that $G[Z]$ is dragon. We define the contracted p-graph on $G / Z$ as follows. Let $Z_{1}$ be the uniquely reachable nodes in $G[Z]$, say a $\in Z_{1}$ is $\omega_{a}$-reachable. Let $A / Z:=A-Z+\{Z\}$. Let $\Omega^{\prime}:=\Omega+\bullet$ for some new element $\bullet \notin \Omega$. Let $\omega_{Z}(s):=\omega(s)$ for all $s \in A / Z-\{Z\}$, and let $\omega_{Z}(\{Z\}):=\bullet$. We define $\pi_{Z}(e)$ by the following case splitting. If $e$ is disjoint from $Z$, then we define $\pi_{Z}(e)$ by extending $\pi(e)$ to $\Omega^{\prime}$ by mapping $\bullet$ to $\bullet$. For an edge ab with $a \in Z_{1}, b \notin Z$ we label its image $\{Z\} b$ s.t. $\pi_{Z}(\{Z\} b)(\{Z\})=\pi(a b)\left(\omega_{a}\right)$. For an edge $a b$ with $a \in Z-Z_{1}, b \notin Z$ we define let $\pi_{Z}(\{Z\} b):=\boldsymbol{J} \boldsymbol{J}$.

We define the contraction of a node-disjoint family $\mathcal{Z}$ of dragons $G / \mathcal{Z}, A / \mathcal{Z}, \omega_{\mathcal{Z}}, \pi_{\mathcal{Z}}$ by contracting the dragons in $\mathcal{Z}$ one-by-one. By definition, a contraction has the following properties.

Claim 4.4 (Expansion of a packing) From any packing in $G / \mathcal{Z}$ one can construct a packing in $G$ which exposes the same number of terminals.

Claim 4.5 (Pre-image of a dragon) The pre-image of a dragon $Z_{1}$ in $G / \mathcal{Z}$ is dragon. (Thus, $\mathcal{Z} / Z_{1}:=$ $\left\{Z: Z \in \mathcal{Z},\{Z\} \notin Z_{1}\right\} \cup\left\{\right.$ the pre-image of $\left.Z_{1}\right\}$ is a finer node-disjoint family of dragons.)

\section{The 3-Way Lemma and the algorithm}

Our main tool in the algorithm is the 3-Way Lemma for packings. Consider a packing $\mathcal{P}$ in $G$ and a dragon $Z$ in $G$. We say $\mathcal{P}$ is equipped with $Z$ if $\mathcal{P}$ consists of some paths disjoint from $V(Z)$ and exactly $\nu(G[Z])=(|A \cap V(Z)|-1) / 2$ paths inside $Z$.

Lemma 5.1 (The 3-way Lemma) Consider a p-graph with a packing $\mathcal{P}$. Then at least one of the following alternatives holds:

1. There is a packing $\mathcal{R}$ with $|\mathcal{R}|=|\mathcal{P}|+1$.

2. There is a packing $\mathcal{R}$ s.t. $|\mathcal{R}|=|\mathcal{P}|$, and is equipped with a rod or an odd cycle.

3. There is a 2-cover $c$ such that $2|\mathcal{P}|=|| c||$. (I.e. a verifying 2-cover for $2 \times \mathcal{P}$ )

The 3-Way Lemma is applied sequentially in the algorithm to construct sequences of contractions. A sequence of contractions is a sequence $\left(\mathcal{Z}_{1}, G_{1}, \mathcal{P}_{1}, \mathcal{R}_{1}, S_{1}\right), \cdots,\left(\mathcal{Z}_{m}, G_{m}, \mathcal{P}_{m}, \mathcal{R}_{m}, S_{m}\right)$, $\left(\mathcal{Z}_{m+1}, G_{m+1}, \mathcal{P}_{m+1}\right)$ with $m \geq 0$, and the following properties. $\mathcal{Z}_{0}=\emptyset$, and $\mathcal{Z}_{i}$ is a node-disjoint family of dragons in $G . G_{i}=\left(V_{i}, E_{i}\right):=G / \mathcal{Z}_{i} . G_{i}\left[S_{i}\right]$ is an odd cycle or a rod, where $S_{i} \subseteq V_{i}$. $\mathcal{R}_{i}$ is a packing in $G_{i}$ which is equipped with $S_{i} . \mathcal{P}_{i+1}:=\mathcal{R}_{i} / S_{i}, \mathcal{Z}_{i+1}:=\mathcal{Z}_{i} / S_{i}$ for $i=1, \cdots, m$. Each $\mathcal{P}_{i}, \mathcal{R}_{i}$ leaves the same number of terminals uncovered.

The proof of Theorem 3.1 and the algorithm relies on the following key observation, which provides a tool to construct a verifying pair. It says that from a 2-packing verification in a contraction we can construct a packing verification in the original p-graph. 
Lemma 5.2 (Constructing a verifying pair) Suppose we have a sequence of contractions, and a 2-cover $c$ in $G_{m+1}$ with $2\left|\mathcal{P}_{m+1}\right|=|| c||$. Then for all $i, \mathcal{P}_{i}$ is a maximum packing in $G_{i}$ and one can construct a verifying pair for $\mathcal{P}_{i}$.

Now we are in position to sketch the algorithm. Our algorithm has an input of a p-graph $G$ and a packing $\mathcal{P}$. The output is either a larger packing, or a verifying pair for $\mathcal{P}$. The algorithm starts off with initiating the trivial sequence of contractions, $m=0$. In a general step, apply Lemma 5.1 to $G_{m+1}, \mathcal{P}_{m+1}$ ! If alternative 1 holds, then by Claim 4.4 one can construct a packing in $G$ larger than $\mathcal{P}$. If alternative 2 holds, then by Claim 4.5 one can construct a longer sequence of contractions. If alternative 3 holds, then by Claim 5.2 $\mathcal{P}$ is maximum, and a verifying pair can be constructed. Full proofs are given in (7). Detailed analysis of the algorithm implies that dragons have a so-called dragon-decomposition.

Definition 5.3 A dragon-decomposition is given by a forest $F \subseteq E$ which has the following properties.

1. The components of forest $(V(F) \cup A, F)$ are exactly $\left\{F_{a}\right.$ : for each $\left.a \in A\right\}$ s.t. for each $a \in A$ we have $A \cap V\left(F_{a}\right)=\{a\}$.

2. Let $\omega^{F}: V(F) \cup A \rightarrow \Omega$ be the (uniquely defined) function s.t. each edge in $F$ is $\omega^{F}$-balanced. Let $F^{\prime}$ be the set of $\omega^{F}$-balanced edges. Let $\mathcal{K}$ is the family of components of $G-F^{\prime} . F / \mathcal{K}$ is a tree.

3. $K, V(F) \cap V(K), \omega^{F}, \pi$ is critical.

Lemma 5.4 Dragons are exactly those p-graphs which have a dragon-decomposition. $V(F) \cup A$ is exactly the set of uniquely reachable nodes.

\section{References}

[1] M. Chudnovsky, J.F. Geelen, B. Gerards, L. Goddyn, M. Lohman, P. Seymour, Packing non-zero A-paths in group-labeled graphs, submitted

[2] M. Chudnovsky, W.H. Cunningham, J.F. Geelen, An algorithm for packing non-zero A-paths in group-labeled graphs, manuscript

[3] T. Gallai, Maximum-minimum Sätze und verallgemeinerte Faktoren von Graphen, Acta Mathematica Academiae Scientarim Hungaricae 12 (1961) 131-137.

[4] L. Lovász, Matroid matching and some applications, Journal of Combinatorial Theory Ser. B 28 (1980) 208-236.

[5] W. Mader, Über die Maximalzahl kreuzungsfreier H-Wege, Archiv der Mathematik (Basel) 31 (1978) 387-402.

[6] G. Pap, Packing non-returning A-paths, submitted

[7] G. Pap, Packings and 2-packings of non-returning A-paths in p-graphs, manuscript

[8] A. Schrijver, A short proof of Mader's $\mathcal{S}$-paths theorem, Journal of Combinatorial Theory Ser. B 85 (2001) 319-321.

[9] A. Sebő, L. Szegő, The path-packing structure of graphs, in: Integer Programming and Combinatorial Optimization (Proceedings of 10th IPCO Conference, New York, 2004; D. Bienstock, G. Nemhauser, eds.) Lecture Notes in Computer Science 3064, Springer-Verlag Berlin Heidelberg (2004) 256-270. 
